\title{
A Novel Approach to the Design of Oversampling Low-Delay Complex-Modulated Filter Bank Pairs
}

\author{
Thomas Kurbiel (EURASIP Member), Heinz G. Göckler (EURASIP Member), \\ and Daniel Alfsmann (EURASIP Member)
}

Digital Signal Processing Group, Ruhr-Universität Bochum, 44780 Bochum, Germany

Correspondence should be addressed to Thomas Kurbiel, kurbiel@nt.ruhr-uni-bochum.de

Received 15 December 2008; Revised 5 June 2009; Accepted 9 September 2009

Recommended by Sven Nordholm

In this contribution we present a method to design prototype filters of oversampling uniform complex-modulated FIR filter bank pairs. Especially, we present a noniterative two-step procedure: (i) design of analysis prototype filter with minimum group delay and approximately linear-phase frequency response in the passband and the transition band and (ii) Design of synthesis prototype filter such that the filter bank pairs distortion function approximates a linear-phase allpass function. Both aliasing and imaging are controlled by introducing sophisticated stopband constraints in both steps. Moreover, we investigate the delay properties of oversampling uniform complex-modulated FIR filter bank pairs in order to achieve the lowest possible filter bank delay. An illustrative design example demonstrates the potential of the design approach.

Copyright (C) 2009 Thomas Kurbiel et al. This is an open access article distributed under the Creative Commons Attribution License, which permits unrestricted use, distribution, and reproduction in any medium, provided the original work is properly cited.

\section{Introduction}

A digital filter bank pair (FBP) is represented by a cascade connection of an analysis filter bank (AFB) for signal decomposition and a synthesis filter bank (SFB) for signal reconstruction. In this contribution, we are exclusively interested in FBP that are most efficient in terms of (i) low power consumption calling for minimum computational load and a modular structure (minimum control overhead), and (ii) low overall ASFB signal (group) delay. The former property is most important if the FBP is part of mobile equipment with tight energy constraints, most pronounced in hearing aids (HAs) [1], while the latter requirement must, for instance, be considered in two-way communication systems or HA, where the total group delay of the FBP shall not exceed 5-8 milliseconds [2,3] to allow for sufficient margin for extensive subband signal processing.

The above computational constraints are best accounted for by using uniform, maximally decimating (criticallysampling), complex-modulated (DFT) polyphase FB applying FIR filters, where all individual frequency responses of the AFB or SFB are frequency-shifted versions of that of the corresponding prototype lowpass filters, respectively, [4-6].
As it is well known, critical sampling in FBP gives rise to severe aliasing in case of low-order (prototype) filters with overlapping frequency responses which, in general, can be compensated for by proper matching of the AFB and SFB (prototype) filters $[5,6]$. In contrast, we are interested in FBP that call for extensive subband signal manipulation, where aliasing compensation approaches cannot be used. As a result, the design of FBP considered in this contribution calls for moderately oversampled subband signals. Thus, nonlinear distortion due to aliasing and imaging can exclusively be controlled by adequate stopband rejection of the respective (prototype) filters. As an example, stopband magnitude constraints for FBP in HA are derived in [7].

When considering linear-phase (LP) FIR filters for the prototype design, the above stringent group delay requirements are best met, if the filter lengths are as small as possible [8]. Essentially the same applies to low-delay FIR filter designs [9].

In the past, many attempts to design FIR lowpass filters with low group delay have been made [9-12]. In [13], a procedure for the design of FIR Nyquist filters with low group delay was proposed that is based on the Remez exchange algorithm. With the mentioned approaches a filter group 
delay can always be obtained that ranges below the group delay of a corresponding LP FIR filter. However, the absolute minimum value of the passband group delay was of no concern. In contrast, for instance, Lang [14] has shown that his algorithms for the constrained design of digital filters with arbitrary magnitude and phase responses have the potential to achieve a considerable reduction of group delay as compared to LP FIR filters, even for high-order FIR filters.

Moreover, several solutions to the problem of low-delay filter banks have been suggested in literature. In [15], an iterative method for the design of oversampling DFT filter banks has been proposed, which allows for controlling the distortion function for each frequency and jointly minimizes aliasing and imaging. The demand for low group delay particularly of the AFB prototype filters has not been asked for explicitly. Based on the algorithm [15] the approach [16] introduces additional constraints to the delay and phase responses. Noncritical decimation has also been suggested in [17], where both filter bank delay aspects and magnitude deviations of the distortion function have not especially been taken into consideration. In [18] the problems of aliasing effect and amplitude distortion are studied. Prototype filters which are optimised with respect to those properties are designed and their performances are compared. Moreover, the effect of the number of subbands, the oversampling factors, and the length of the prototype filter are also studied. Using the multicriteria formulation, all Pareto optimums are sought via the nonlinear programming technique. In [19] a hybrid optimization method is proposed to find the Pareto optimums of this highly nonlinear problem. Furthermore it is shown that Kaiser and Dolph-Chebyshev windows give the best overall performance with or without oversampling.

From a filter bank system theoretic point of view, we pursue three objectives, representing steps towards the design of oversampling uniform complex-modulated (FFT) FIR filter bank pairs (FBPs) allowing for extensive subband signal manipulation. We restrict ourselves to integer oversampling factors as defined by

$$
\mathcal{O}=\frac{I}{M} \in \mathbb{N}, \quad \mathcal{O}>1
$$

in order not to constrain the applicability of polyphase prototype filters in any form $[4,6]$, where $I$ represents the number of FB channels and $M \in \mathbb{N}$ the common decimation or interpolation factor of the FBP, respectively.

(1) In Section 2 being related to the first objective of this paper, we begin with a system-theoretic description and analysis of oversampling $I$-channel complex-modulated FIR filter bank pairs without subband signal manipulation, which supplements and extends the results reported in [16]. In particular, we investigate the properties of the distortion function $[5,6]$, the overall single-input single-output (SISO) transfer function of the filter bank pair that ideally approximates a linear-phase allpass function. We show that both the magnitude and the group delay of the FBP distortion function are periodic versus frequency. Furthermore, the group delay of the FBP is investigated in detail.

(2) For a first design step (cf. Section 3), we introduce a novel procedure for the constrained design of lowdelay narrow-band FIR prototype filters for oversampling complex-modulated filter banks with an approximately linear-phase frequency response in the passband and the transition bands. As the objective function to be minimised we adopt a particular representation of the group delay [20], while the stopband magnitude specifications of the prototype filter, as derived in [7], serve as constraints to control subband signal aliasing or imaging, respectively. In the first design step this procedure is used either for the design of the SFB or the AFB prototype filter.

(3) For the second and final design step (cf. Section 4), we use the deviation of the FBP distortion function from unity as the objective function. To this end, the AFB (or SFB) FIR prototype filter is designed subject to the stopband magnitude constraints, as given by [7], while the SFB (or AFB) prototype filter is fixed to the design obtained in the previous step. By this procedure the AFB and SFB prototype filters' magnitude responses are matched in the passbands and the transition bands without further consideration of the overall FBP group delay, aiming at minimum, possibly differing AFB and SFB prototype filter orders.

To illustrate the results and the potential of the design procedure, we present a design example in Section 5. Finally, in Section 6 we draw some conclusions.

\section{Oversampling Complex-Modulated FIR Filter Bank Pairs}

We begin with the introduction of our filter bank notation and a system-theoretic description and analysis of uniform oversampling I-channel complex-modulated FIR filter bank pairs (FBP) without subband signal manipulation, the principle of which is shown in Figure 1. In particular, we investigate the properties of the distortion function [5, 6], which is required to approximate a linear-phase allpass function.

2.1. Distortion Function. For a uniform oversampling complex-modulated $I$-channel FBP the AFB und SFB filters, respectively, are derived from common prototype filters $[5,6]$ :

$$
\begin{array}{ll}
H_{l}\left(z_{\mathrm{i}}\right)=H\left(z_{\mathrm{i}} W_{I}^{l}\right), & l=0, \ldots, I-1, \\
G_{l}\left(z_{\mathrm{i}}\right)=G\left(z_{\mathrm{i}} W_{I}^{l}\right), \quad l=0, \ldots, I-1 .
\end{array}
$$




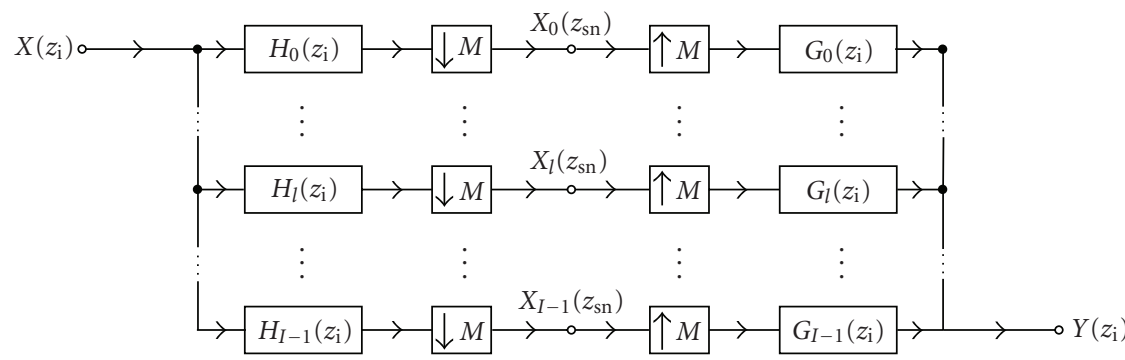

FIGURE 1: Uniform oversampling filter bank pair, oversampling factor $\mathcal{O}=I / M \in \mathbb{N}$.

Both prototype filters are real-valued FIR filters represented by their causal transfer functions:

$$
\begin{aligned}
& H\left(z_{\mathrm{i}}\right)=\sum_{n=0}^{N_{\mathrm{h}}-1} h(n) \cdot z_{\mathrm{i}}^{-n}=\mathbf{c}_{\mathrm{h}}^{\mathrm{T}}\left(z_{\mathrm{i}}\right) \cdot \mathbf{h}, \\
& G\left(z_{\mathrm{i}}\right)=\sum_{n=0}^{N_{\mathrm{g}}-1} g(n) \cdot z_{\mathrm{i}}^{-n}=\mathbf{c}_{\mathrm{g}}^{\mathrm{T}}\left(z_{\mathrm{i}}\right) \cdot \mathbf{g},
\end{aligned}
$$

where the vectors

$$
\begin{aligned}
& \mathbf{h}=\left[h(0), h(1), \ldots, h\left(N_{\mathrm{h}}-1\right)\right]^{\mathrm{T}} \in \mathbb{R}^{N_{\mathrm{h}}}, \\
& \mathbf{g}=\left[g(0), g(1), \ldots, g\left(N_{\mathrm{g}}-1\right)\right]^{\mathrm{T}} \in \mathbb{R}^{N_{\mathrm{g}}}
\end{aligned}
$$

contain $N_{\mathrm{h}}$ or $N_{\mathrm{g}}$ coefficients of the impulse response, respectively, and the vectors

$$
\begin{aligned}
& \mathbf{c}_{\mathrm{h}}\left(z_{\mathrm{i}}\right)=\left[1, z_{\mathrm{i}}^{-1}, z_{\mathrm{i}}^{-2}, \ldots, z_{\mathrm{i}}^{-\left(N_{\mathrm{h}}-1\right)}\right]^{\mathrm{T}}, \\
& \mathbf{c}_{\mathrm{g}}\left(z_{\mathrm{i}}\right)=\left[1, z_{\mathrm{i}}^{-1}, z_{\mathrm{i}}^{-2}, \ldots, z_{\mathrm{i}}^{-\left(N_{\mathrm{g}}-1\right)}\right]^{\mathrm{T}}
\end{aligned}
$$

the associated delays.

The input signal $x(n) \stackrel{\mathrm{zT}}{\leftrightarrow} X\left(z_{\mathrm{i}}\right)$ in Figure 1 is simultaneously passed through all AFB channel filters $H_{l}\left(z_{\mathrm{i}}\right), l=$ $0, \ldots, I-1$ and subsequently downsampled by a factor of $M$, yielding

$$
\begin{array}{r}
X_{l}\left(z_{\mathrm{sn}}\right)=\frac{1}{M} \sum_{k=0}^{M-1} H_{l}\left(z_{\mathrm{sn}}^{1 / M} W_{M}^{k}\right) X\left(z_{\mathrm{sn}}^{1 / M} W_{M}^{k}\right) \\
l=0,1, \ldots, I-1,
\end{array}
$$

using the alias component representation $[5,6]$, and $W_{M}=$ $\mathrm{e}^{-\mathrm{j} 2 \pi / M}$. In the SFB, the $M$-fold upsampled subband signals $X_{l}\left(z_{\mathrm{i}}^{M}\right)=X_{l}\left(z_{\mathrm{sn}}\right)$ are combined to form the $z$-domain output signal representation [6]:

$$
Y\left(z_{\mathrm{i}}\right)=\sum_{l=0}^{I-1} G_{l}\left(z_{\mathrm{i}}\right) X_{l}\left(z_{\mathrm{i}}^{M}\right)
$$

Inserting the upsampled form of (7) into (8), with $z_{\mathrm{sn}}=z_{\mathrm{i}}^{M}$ we obtain

$$
\begin{aligned}
Y\left(z_{\mathrm{i}}\right) & =\sum_{l=0}^{I-1} G_{l}\left(z_{\mathrm{i}}\right)\left[\frac{1}{M} \sum_{k=0}^{M-1} H_{l}\left(z_{\mathrm{i}} W_{M}^{k}\right) X\left(z_{\mathrm{i}} W_{M}^{k}\right)\right] \\
& =\frac{1}{M} \sum_{k=0}^{M-1} X\left(z_{\mathrm{i}} W_{M}^{k}\right)\left[\sum_{l=0}^{I-1} H_{l}\left(z_{\mathrm{i}} W_{M}^{k}\right) G_{l}\left(z_{\mathrm{i}}\right)\right] .
\end{aligned}
$$

Obviously the output signal representation $Y\left(z_{\mathrm{i}}\right)$ depends on all $M$ modulation components $X\left(z_{\mathrm{i}} W_{M}^{k}\right), k=0, \ldots, M-1$, of the input signal. All these components are filtered by the compound term $\sum_{l=0}^{I-1} H_{l}\left(z_{\mathrm{i}} W_{M}^{k}\right) G_{l}\left(z_{\mathrm{i}}\right)$ and eventually combined. The transfer function of the zeroth $(k=0)$ modulation component is generally denoted as the distortion function $[5,6]$ :

$$
F_{\text {dist }}\left(z_{\mathrm{i}}\right)=\frac{1}{M}\left[\sum_{l=0}^{I-1} H_{l}\left(z_{\mathrm{i}}\right) G_{l}\left(z_{\mathrm{i}}\right)\right] .
$$

In our approach this distortion function determines the properties of the FBP almost exclusively, since aliasing and imaging is assumed to be negligible as a result of sufficiently high AFB and SFB prototype filter stopband attenuation. Inserting (3) and (4) into (10), we obtain

$$
F_{\text {dist }}\left(z_{\mathrm{i}}\right)=\frac{1}{M}\left[\sum_{l=0}^{I-1} H\left(z_{\mathrm{i}} W_{I}^{l}\right) G\left(z_{\mathrm{i}} W_{I}^{l}\right)\right] .
$$

Next, the properties of the distortion function (11) are investigated in the time-domain.

2.2. Time-Domain Analysis. In this section we present a novel time-domain interpretation of the distortion function. We begin with introducing the FBP impulse response

$$
s(n)=h(n) * g(n) \stackrel{\mathrm{zT}}{\longleftrightarrow} S\left(z_{\mathrm{i}}\right)=H\left(z_{\mathrm{i}}\right) \cdot G\left(z_{\mathrm{i}}\right) .
$$

The length of $s(n)$ is given by $[8,20]$

$$
N_{\mathrm{s}}=N_{\mathrm{h}}+N_{\mathrm{g}}-1 \text {. }
$$

The distortion function (11) is reformulated by introducing (12) and by using (1):

$$
F_{\text {dist }}\left(z_{\mathrm{i}}\right)=\frac{\mathcal{O}}{I} \sum_{l=0}^{I-1} S\left(z_{\mathrm{i}} W_{I}^{l}\right)=\mathcal{O} \cdot S_{0}^{(I)}\left(z_{\mathrm{i}}^{I}\right) .
$$


In time-domain this relation is expressed as

$$
f_{\text {dist }}(n)=\mathcal{O} \cdot s_{0}^{(I)}(n)= \begin{cases}\mathcal{O} \cdot s(m I) & \forall n=m I, m \in \mathbb{N}, \\ 0 & \forall n \neq m I, m \in \mathbb{N} .\end{cases}
$$

As a result the distortion function represents the $z$-transform of the zeroth polyphase component of $s(n)[5,6]$. Considering (13) and (15) the distortion function in time-domain $f_{\text {dist }}(n)$ has only $\left\lfloor\left(N_{s}-1\right) / I\right\rfloor+1$ nonzero terms, which leads to the $z$-domain representation of the distortion function:

$$
F_{\text {dist }}\left(z_{\mathrm{i}}\right)=\mathcal{O} \sum_{m=0}^{\left\lfloor\left(N_{\mathrm{s}}-1\right) / I\right\rfloor+1} s(m I) \cdot z_{\mathrm{i}}^{-m I},
$$

where all zero values of the FBP impulse response $s(n)$ according to (15) have been discarded. Hence, the corresponding discrete-time Fourier transform of (16) is $2 \pi / I-$ periodic, where $I$ is the number of FBP channels. As a consequence, both the magnitude response and the group delay response of the FBP distortion function possess this $2 \pi / I$-periodicity property.

2.3. Potential Delays. Next, we show that the mean group delay of a uniform $I$-channel complex-modulated oversampling FBP is restricted to integer multiples of the number $I$ of FBP channels. This is an inherent characteristic of a complex-modulated filter bank and has first been shown in [16]. Exploiting the above $2 \pi / I$-periodicity, we define the mean group delay:

$$
\bar{\tau}_{\mathrm{g}}^{\mathrm{dist}}=\frac{I}{2 \pi} \int_{-\pi / I}^{\pi / I} \tau_{\mathrm{g}}^{\mathrm{dist}}\left(\Omega^{(\mathrm{i})}\right) d \Omega^{(\mathrm{i})} .
$$

Since the distortion function (16) is conjugate symmetric:

$$
\begin{aligned}
{\left[F_{\text {dist }}\left(\mathrm{e}^{-\mathrm{j} \Omega^{(\mathrm{i})}}\right)\right]^{*} } & =\left[\mathcal{O} \sum_{m=0}^{\left\lfloor\left(N_{s}-1\right) / I\right\rfloor} s(m I) \cdot \mathrm{e}^{\mathrm{j} m I \Omega^{(\mathrm{i})}}\right]^{*} \\
& =F_{\text {dist }}\left(\mathrm{e}^{\mathrm{j} \Omega^{(\mathrm{i})}}\right)
\end{aligned}
$$

the group delay of the filter bank is an even function allowing for the modification of (17):

$$
\bar{\tau}_{\mathrm{g}}^{\mathrm{dist}}=\frac{I}{\pi} \int_{0}^{\pi / I} \tau_{\mathrm{g}}^{\mathrm{dist}}\left(\Omega^{(\mathrm{i})}\right) d \Omega^{(\mathrm{i})} .
$$

Using the definition of the group delay as the negative derivation of the phase $\varphi\left(\Omega^{(i)}\right)$ :

$$
\tau_{g}\left(\Omega^{(\mathrm{i})}\right)=-\frac{\mathrm{d} \varphi\left(\Omega^{(\mathrm{i})}\right)}{\mathrm{d} \Omega^{(\mathrm{i})}},
$$

it follows from (19) that

$$
\bar{\tau}_{\mathrm{g}}^{\mathrm{dist}}=-\frac{I}{\pi}\left[\varphi\left(\Omega^{(\mathrm{i})}\right)\right]_{0}^{\pi / I}=\frac{I}{\pi}\left[\varphi(0)-\varphi\left(\frac{\pi}{I}\right)\right] .
$$

To determine the phase response at $\Omega^{(\mathrm{i})}=0$ and $\Omega^{(\mathrm{i})}=\pi / I$ we use (16) for $z_{\mathrm{i}}=\mathrm{e}^{\mathrm{j} 0}=1$ :

$$
F_{\text {dist }}\left(\mathrm{e}^{\mathrm{j} 0}\right)=\mathcal{O} \sum_{m=0}^{\left\lfloor\left(N_{\mathrm{s}}-1\right) / I\right\rfloor+1} s(m I) \in \mathbb{R}
$$

which is real valued according to (12) since we only consider real analysis and synthesis prototype filters. Moreover, the magnitude of the distortion function is supposed to be approximately unity, $F_{\text {dist }}\left(\mathrm{e}^{\mathrm{j} 0}\right) \approx 1 \cdot \mathrm{e}^{\mathrm{j} \varphi_{\text {dist }}(0)} \in \mathbb{R}$; therefore the phase response at zero frequency is

$$
\varphi_{\text {dist }}(0)=\kappa_{1} \cdot \pi, \quad \kappa_{1} \in \mathbb{Z} .
$$

With the same considerations for $z_{\mathrm{i}}^{I}=\mathrm{e}^{\mathrm{j}(\pi / I) I}=-1$, we get

$$
F_{\text {dist }}\left(\mathrm{e}^{\mathrm{j}(\pi / I)}\right)=\mathcal{O} \sum_{m=0}^{\left\lfloor\left(N_{\mathrm{s}}-1\right) / I\right\rfloor+1} \mathrm{~s}(m I) \cdot(-1)^{m} \in \mathbb{R} .
$$

Since at this frequency the distortion function is again realvalued and approximately unity, $F_{\text {dist }}\left(\mathrm{e}^{\mathrm{j}(\pi / I)}\right) \approx 1 \cdot \mathrm{e}^{\mathrm{j} \varphi_{\text {dist }}(\pi / I)} \in$ $\mathbb{R}$, we conclude that

$$
\varphi_{\text {dist }}\left(\frac{\pi}{I}\right)=\kappa_{2} \cdot I, \quad \kappa_{2} \in \mathbb{Z} .
$$

Combining the results (23) and (25) with (21) yields

$$
\bar{\tau}_{\mathrm{g}}^{\mathrm{dist}}=\frac{I}{\pi}\left[\kappa_{1} \cdot \pi-\kappa_{2} \cdot \pi\right]=\left(\kappa_{1}-\kappa_{2}\right) \cdot I .
$$

The result states that a complex-modulated filter bank can only approximate delays of the form $\tau_{\mathrm{g}}=\kappa \cdot I, \kappa \in \mathbb{Z}$.

Finally, we present a system-theoretic interpretation of the fact that the overall group delay is restricted to $\tau_{\mathrm{g}}=\kappa \cdot I$. In the following all examinations of the distortion function are performed in time-domain using $f_{\text {dist }}(n)$. According to (15) the distortion function represents the zeroth polyphase component of $s(n)=h(n) * g(n)$ with the prototype filters (3) and (4). Therefore $f_{\text {dist }}(n)$ has only $\left\lfloor\left(N_{s}-1\right) / I\right\rfloor+1$ nonzero terms which are located at indices that are integral multiples of $I$.

We begin with an ideal distortion function of constant magnitude response and linear-phase. Since the distortion function in time-domain can be seen as the impulse response of an FIR filter, the upper demand is equivalent to the demand for an FIR allpass. According to the theory of FIR filters this can only be achieved by a simple delay $[8,20,21]$. Therefore all the nonzero terms of $f_{\text {dist }}(n)$ have to be zero except for one. The resulting distortion function is

$$
F_{\text {dist }}\left(z_{\mathrm{i}}\right)=z_{\mathrm{i}}^{-d I}, \quad d \in \mathbb{N} .
$$

Hence, under ideal allpass conditions, the delay of a uniform complex-modulated FBP is restricted to integer multiples of the number $I$ of channels.

Next we relieve the demand for exactly constant magnitude response and ask only for exactly linear-phase. The nonzero terms of $f_{\text {dist }}(n)$ must exhibit a symmetry in order to impose a linear-phase distortion function. For illustration, 
we start with a simple example assuming an odd length: $\left\lfloor\left(N_{\mathrm{s}}-1\right) / I\right\rfloor+1=3$. To gain a better overview the nonzeros terms of $f_{\text {dist }}(n)$ are put into a vector:

$$
\mathbf{f}_{\text {dist }}=[\varepsilon, 1, \varepsilon]^{\mathrm{T}},
$$

where $\varepsilon \ll 1$ is provided. The distortion function is the discrete-time Fourier transform of the upper expression:

$$
\begin{aligned}
F_{\text {dist }}\left(\mathrm{e}^{\mathrm{j} \Omega^{(\mathrm{i})}}\right) & =\varepsilon+\mathrm{e}^{-\mathrm{j} \Omega^{(\mathrm{i})} I}+\varepsilon \cdot \mathrm{e}^{-\mathrm{j} 2 \Omega^{(\mathrm{i})} I} \\
& =\mathrm{e}^{-\mathrm{j} \Omega^{(\mathrm{i})} I}\left[1+2 \cdot \varepsilon \cdot \cos \left(\Omega^{(\mathrm{i})} I\right)\right] .
\end{aligned}
$$

As a result, the constant group delay of

$$
\tau_{\mathrm{g}}^{\min }=I \cdot \frac{\left\lfloor\left(N_{\mathrm{s}}-1\right) / I\right\rfloor-1}{2}=I
$$

is obtained, while the magnitude response of (16) varies in the vicinity of

$$
1-2 \varepsilon \leq\left|F_{\text {dist }}\left(\mathrm{e}^{\mathrm{j} \Omega^{(\mathrm{i})}}\right)\right| \leq 1+2 \varepsilon .
$$

Since $\varepsilon \ll 1$, the distortion function approximates a linearphase allpass function sufficiently well. Similar results can be obtained with any even order $\left\lfloor\left(N_{s}-1\right) / I\right\rfloor$ (odd length) of the downsampled distortion function (16). From the theory of linear-phase FIR filters it is well known [8, 20,21] that the zero-phase frequency responses of even-length symmetric FIR filters always possess at least a single zero at $f=f_{\mathrm{i}} / I$ $\left(z_{\mathrm{i}}^{I}=-1\right)$. All antimetric linear-phase FIR filters are likewise unusable, since they have zero transfer at zero frequency $\left(z_{\mathrm{i}}^{I}=1\right)$. Hence, in case of exactly linear-phase distortion functions, the impulse response is restricted to even order, to positive symmetry, and the only possible group delay is given by (30).

Finally we relieve the demand for exactly linear-phase and ask only for approximately constant magnitude response and approximately linear-phase. Thus $f_{\text {dist }}(n)$ is no longer restricted to be symmetric. As a result, the position $d \cdot I$ of the dominating coefficient of the distortion function in time-domain can again take on any value according to $d \in\left\{1,2, \ldots,\left\lfloor\left(N_{\mathrm{s}}-1\right) / I\right\rfloor\right\}$, while all other coefficients at positions $m \neq d \in\left\{1,2, \ldots,\left\lfloor\left(N_{s}-1\right) / I\right\rfloor\right\}$ must be kept close to zero by optimisation. Hence, the overall mean delay of a uniform oversampling complex-modulated FBP results in

$$
\tau_{\mathrm{g}}=d \cdot I
$$

Note that the above considerations of linear-phase FIR filters likewise apply approximately.

\section{Design of Low-Delay FIR Prototype Filter}

In this section, we develop a procedure for the design of real-valued narrowband FIR lowpass prototype filters for the AFB. We are aiming at (i) minimum group delay both in the pass and in the transition band and (ii) meeting tight magnitude frequency response constraints for the stopband. The requirements concerning the stopband attenuation can vary with each frequency. Especially, we look for a unique solution that yields the globally optimum design. To this end, we introduce for the first time a convex objective function for group delay minimisation, whereas the magnitude requirements are used as design constraints.

3.1. Objective Function. Subsequently, a convex objective function for group delay minimisation of narrowband FIR filters is developed that delivers the desired globally optimum design result. To begin with, let us use the polar coordinate representation of (3):

$$
H\left(\mathrm{e}^{\mathrm{j} \Omega^{(\mathrm{i})}}\right)=\left|H\left(\mathrm{e}^{\mathrm{j} \Omega^{(\mathrm{i})}}\right)\right| \cdot \mathrm{e}^{\mathrm{j} \varphi\left(\Omega^{(\mathrm{i})}\right)},
$$

where $\varphi\left(\Omega^{(i)}\right)$ describes the phase of the FIR filter frequency response [20].

By calculating the first derivative of the frequency response as given by both (33) and (13) with respect to the normalised frequency $\Omega^{(i)}$, we obtain a relation that contains the group delay in one of its summands:

$$
\begin{aligned}
\mathrm{j} \frac{d H\left(\mathrm{e}^{\mathrm{j} \Omega^{(\mathrm{i})}}\right)}{d \Omega^{(\mathrm{i})}}= & \tau_{g}\left(\Omega^{(\mathrm{i})}\right) \cdot H\left(\mathrm{e}^{\mathrm{j} \Omega^{(\mathrm{i})}}\right) \\
& +\mathrm{j} \cdot \frac{d\left|H\left(\mathrm{e}^{\mathrm{j} \Omega^{(\mathrm{i})}}\right)\right|}{d \Omega^{(\mathrm{i})}} \cdot \mathrm{e}^{\mathrm{j} \varphi\left(\Omega^{(\mathrm{i})}\right)} .
\end{aligned}
$$

Note that (34) is equivalently represented in time-domain according to the differentiation in frequency property of the discrete-time Fourier transform:

$$
h_{\text {deriv }}(n)=n \cdot h(n) \stackrel{\text { DTFT }}{\longleftrightarrow} \frac{d H\left(\mathrm{e}^{\mathrm{j} \Omega^{(\mathrm{i})}}\right)}{d \Omega^{(\mathrm{i})}} .
$$

Next we apply the generalized Parseval's theorem which is [8]

$$
\sum_{n=-\infty}^{\infty} x(n) y^{*}(n)=\frac{1}{2 \pi} \int_{-\pi}^{\pi} X\left(\mathrm{e}^{\mathrm{j} \Omega^{(\mathrm{i})}}\right) Y^{*}\left(\mathrm{e}^{\mathrm{j} \Omega^{(\mathrm{i})}}\right) d \Omega^{(\mathrm{i})} .
$$

On the left side of (36) we substitute $x(n)=h_{\text {deriv }}(n)=n$. $h(n)$ and $y(n)=h(n)$. On the right side the corresponding terms in the frequency domain are inserted. Please note that $X\left(\mathrm{e}^{\mathrm{j} \Omega^{(\mathrm{i})}}\right)$ corresponds to $(33)$. We get

$$
\begin{aligned}
& \sum_{n=0}^{N_{\mathrm{h}}-1} n \cdot h(n)^{2} \\
& \quad=\frac{1}{2 \pi} \int_{-\pi}^{\pi}\left(\tau_{g}\left(\Omega^{(\mathrm{i})}\right) \cdot\left|H\left(\mathrm{e}^{\mathrm{j} \Omega^{(\mathrm{i})}}\right)\right|^{2}\right. \\
& \left.\quad+\mathrm{j} \cdot \frac{d\left|H\left(\mathrm{e}^{\mathrm{j} \Omega^{(\mathrm{i})}}\right)\right|}{d \Omega^{(\mathrm{i})}} \cdot\left|H\left(\mathrm{e}^{\mathrm{j} \Omega^{(\mathrm{i})}}\right)\right|\right) d \Omega^{(\mathrm{i})} .
\end{aligned}
$$

Using the fact that the derivation of even function yields uneven function the integral over the imaginary part of the 
integrand in (37) is zero since the integration interval is symmetric:

$$
\sum_{n=0}^{N_{\mathrm{h}}-1} n \cdot h(n)^{2}=\frac{1}{2 \pi} \int_{-\pi}^{\pi} \tau_{g}\left(\Omega^{(\mathrm{i})}\right) \cdot\left|H\left(\mathrm{e}^{\mathrm{j} \Omega^{(\mathrm{i})}}\right)\right|^{2} d \Omega^{(\mathrm{i})} .
$$

Obviously the rather sophisticated integral corresponds in time-domain to a simple sum. This formula was first introduced in [20].

Next, we proof that (38) posseses all the characteristics the objective function was asked for in last section. This is best shown by examining the following theoretical constrained optimization problem:

$$
\begin{aligned}
& \min _{\mathbf{h}} \frac{1}{2 \pi} \int_{-\pi}^{\pi} \tau_{g}\left(\Omega^{(\mathrm{i})}, \mathbf{h}\right) \cdot\left|H\left(\mathrm{e}^{\mathrm{j} \Omega^{(\mathrm{i})}}, \mathbf{h}\right)\right|^{2} d \Omega^{(\mathrm{i})}, \\
& \text { s.t. } \forall \mathbf{h} \in X,
\end{aligned}
$$

where $X$ is supposed to be the set of all lowpass filters of length $N$ with a distinctive passband (i.e., negligible ripple) and very narrow transition band. Moreover low-pass filters in $X$ are supposed to have a high stopband attenuation. The set $X$ allows us to simplify the right side of (38) and makes it possible to explain its functionality. Due to the second power of the magnitude frequency response and the assumed high stopband attenuation of the filters in $X$ the integrand $\tau_{g}\left(\Omega^{(\mathrm{i})}\right) \cdot\left|H\left(\mathrm{e}^{\mathrm{j} \Omega^{(\mathrm{i})}}\right)\right|^{2}$ is nearly zero in the stopband. The magnitude frequency response is in consequence of the negligible ripple nearly one throughout the passband. And finally due to the assumed very narrow transition band (39) can be simplified in the following way:

$$
\begin{aligned}
& \min _{\mathbf{h}} \frac{1}{2 \pi} \int_{0}^{\Omega_{d}^{(\mathrm{i})}} \tau_{g}\left(\Omega^{(\mathrm{i})}\right) d \Omega^{(\mathrm{i})}, \\
& \text { s.t. } \forall \mathbf{h} \in X .
\end{aligned}
$$

It is evident as seen in (40) that by minimizing the objective function the area bounded by the group delay in the passband is minimized. Minimizing the area results in minimizing the group delay itself in the passband, which is our main purpose. Moreover minimizing the area beneath the group delay yields a smoothing effect. In the stopband the group delay is apparently not minimized at all. Therefore the stopband can be regarded as a "do not care" region thus increasing the available degrees of freedom. Next we look at more realistic filters which do not exhibit negligible transition bands. In this case the second power of the magnitude frequency response in (39) acts in the transition band as a real-valued weighting function for the group delay. Thus guaranteeing that in the transition band close to the passband edge the group delay is minimized in the most prevalent form and close to the stopband edge in the least.

One of the objective function's strongest points is the simple formulation in time-domain as seen in (39). The sum on the left side can readily be expressed by a quadratic form:

$$
\sum_{n=0}^{N_{\mathrm{h}}-1} n \cdot h(n)^{2}=\mathbf{h}^{\mathrm{T}} \cdot \mathbf{D}_{N_{\mathrm{h}}} \cdot \mathbf{h}
$$

The $N_{\mathrm{h}} \times N_{\mathrm{h}}$ diagonal matrix $\mathbf{D}_{N}$ has the following form:

$$
\mathbf{D}_{N_{\mathrm{h}}}=\operatorname{diag}\left(0,1, \ldots, N_{\mathrm{h}}-1\right)=\left(\begin{array}{cccc}
0 & 0 & \cdots & 0 \\
0 & 1 & \cdots & 0 \\
\vdots & \vdots & \ddots & \vdots \\
0 & 0 & \cdots & N-1
\end{array}\right)
$$

This matrix is positive semidefinite, which implies the convexity of the objective function. Hence gradient and Hessian matrix, both important for search methods, can be obtained very easily.

3.2. Constraints. In this section we present functions to set up constraints for the optimization problem. These functions enable us to meet the given magnitude frequency response specifications during the optimization. We show that all functions are convex and in combination with the introduced convex objective function yield a convex optimization problem.

3.2.1. Passband. Narrow-band low-pass filters usually do not exhibit a distinctive passband. In order to obtain a narrowband low-pass filters it is sufficient to ask for

$$
\left|H\left(\mathrm{e}^{\mathrm{j} \Omega^{(\mathrm{i})}}, \mathbf{h}\right)\right|_{\Omega^{(\mathrm{i})}=0}=1,
$$

which is accomplished by formulating an equality constraint. Using the relation $H\left(\mathrm{e}^{\mathrm{j} 0}, \mathbf{h}\right)= \pm\left|H\left(\mathrm{e}^{\mathrm{j} 0}, \mathbf{h}\right)\right|$, whereas the minus sign can be understood as a special case only [8], we reformulate the upper constraint function by using (3) evaluated at $\Omega^{(i)}=0$ :

$$
H\left(\mathrm{e}^{\mathrm{j} 0}\right)=\mathbf{c}_{\mathrm{h}}^{\mathrm{T}}\left(\mathrm{e}^{\mathrm{j} 0}\right) \cdot \mathbf{h} .
$$

The vector $\mathbf{e}^{*}(0)$ is equivalent to the one-vector which is defined as follows: $1:=(1, \ldots, 1)^{\mathrm{T}}$. The linear (referring to h) equality constraint for the passband can thus be stated as follows:

$$
\mathbf{1}^{\mathrm{T}} \cdot \mathbf{h}=1
$$

Since term (44) is a linear function in $\mathbf{h}$, the convexity of the search space defined by the constraints is ensured.

3.2.2. Stopband. The magnitude frequency response specifications in the stopband are defined by a tolerance mask. To this end a nonnegative tolerance value function $\Delta\left(\Omega^{(i)}\right) \geq 0$ is defined, which determines the allowed maximum deviation:

$$
\left|H\left(\mathrm{e}^{\mathrm{j} \Omega^{(\mathrm{i})}}\right)\right| \leq \Delta\left(\Omega^{(\mathrm{i})}\right) \quad \forall \Omega^{(\mathrm{i})} \in \mathbb{B}_{\mathrm{s}} .
$$

The tolerance mask is defined on the region of support $\mathbb{B}_{s}$, the conjunction of all stopbands, which is a subset of the bounded interval $[0, \pi]$. This makes allowances for the symmetry of the frequency response of real-valued filters [8]. Regions of the bounded interval $[0, \pi]$ where no tolerance 
mask is defined are called "do not care" regions. The definition of the tolerance value function $\Delta\left(\Omega^{(\mathrm{i})}\right)$ according to (46) can be used to formulate the remaining constraints. By using the following relation between the magnitude and the real part of a complex number $z_{\mathrm{i}}$, also known as the real rotation theorem $[15,16]$,

$$
\begin{array}{r}
\left|z_{\mathrm{i}}\right|=\max _{\theta \in[0,2 \pi)}\left[\operatorname{Re}\left\{z_{\mathrm{i}} \cdot \mathrm{e}^{-\mathrm{j} \theta}\right\}\right] \geq \\
\quad \operatorname{Re}\left\{z_{\mathrm{i}} \cdot \mathrm{e}^{-\mathrm{j} \theta}\right\}, \\
\forall \theta \in[0,2 \pi),
\end{array}
$$

and applying it for the magnitude frequency response we obtain

$$
\begin{aligned}
\left|H\left(\mathrm{e}^{\mathrm{j} \Omega^{(\mathrm{i})}}, \mathbf{h}\right)\right| & \geq \operatorname{Re}\left\{H\left(\mathrm{e}^{\mathrm{j} \Omega^{(\mathrm{i})}}, \mathbf{h}\right) \cdot \mathrm{e}^{-\mathrm{j} \theta}\right\} \\
& =\sum_{n=0}^{N-1} h(n) \cdot \cos \left(\Omega^{(\mathrm{i})} \cdot n+\theta\right), \quad \forall \theta \in[0,2 \pi) .
\end{aligned}
$$

This term again is a linear function in $\mathbf{h}$ and can be written down using the vector representation as follows:

$$
\left|H\left(e^{j \Omega^{(\mathrm{i})}}, \mathbf{h}\right)\right| \geq \mathbf{c}^{\mathrm{T}}\left(\Omega^{(\mathrm{i})}, \theta\right) \cdot \mathbf{h}, \quad \forall \theta \in[0,2 \pi),
$$

where

$$
\begin{aligned}
& \mathbf{c}\left(\Omega^{(\mathrm{i})}, \theta\right) \\
& \quad=\left[\cos (\theta), \cos \left(\Omega^{(\mathrm{i})}+\theta\right), \ldots, \cos \left([N-1] \cdot \Omega^{(\mathrm{i})}+\theta\right)\right]^{\mathrm{T}},
\end{aligned}
$$

depends not only on the frequency $\Omega$ but on also the additional value $\theta$ as well. Using (49) the inequality constraint can be stated as follows:

$$
\mathbf{c}^{\mathrm{T}}\left(\Omega^{(\mathrm{i})}, \theta\right) \cdot \mathbf{h} \leq \Delta\left(\boldsymbol{\Omega}^{(\mathrm{i})}\right), \quad \forall \boldsymbol{\Omega}^{(\mathrm{i})} \in \mathbb{B}_{\mathrm{s}}, \theta \in[0,2 \pi) .
$$

We see that the region defined by the upper inequality constraint is convex due to the linearity of the left term in h. Please note that the number of constraints in the stopband in the original formulation is infinite regarding to the frequency $\Omega^{(i)}$. In the linearized version according to (51) a second infinite parameter $\theta$ appears, which is induced by the real rotation theorem. Thus the constraints are now infinite regarding both $\Omega^{(\mathrm{i})}$ and $\theta$.

3.3. Constrained Optimization Problem. In this section the convex objective function (41) and the convex constraints (45) and (51) are used to build up a convex constrained optimization problem. Since all used constraint functions are linear in $\mathbf{h}$, the so-called Constraint Qualification is always maintained. The problem can readily be formulated in the following way:

$$
\begin{array}{ll}
\min _{\mathbf{h}} \mathbf{h}^{\mathrm{T}} \cdot \mathbf{D}_{N_{\mathbf{h}}} \cdot \mathbf{h} & \\
\text { s.t. } \mathbf{1}^{\mathrm{T}} \cdot \mathbf{h}=1, \quad \forall \Omega^{(\mathrm{i})}=0 & \\
\mathbf{c}^{\mathrm{T}}\left(\Omega^{(\mathrm{i})}, \theta\right) \cdot \mathbf{h} \leq \Delta\left(\Omega^{(\mathrm{i})}\right), & \forall \Omega^{(\mathrm{i})} \in \mathbb{B}_{s}, \\
& \forall \theta \in[0,2 \pi) .
\end{array}
$$

Due to the fact that the objective function is a quadratic function and the number of constraints is infinite, the overall optimization problem is called convex quadratic semi-infinite optimization problem. The term semi-infinite implies a finite number of unknowns $\mathbf{h}$ yet a infinite number of constraints.

To obtain a computable algorithm the number of constraints has to be reduced to a finite number. The mere discretization of $\Omega^{(i)}$ in the following way:

$$
\Omega_{k}^{(\mathrm{i})}=\frac{\pi}{N_{\mathrm{FFT}}} \cdot k, \quad k=0,1, \ldots, N_{\mathrm{FFT}}
$$

is not sufficient for obtaining a finite optimization problem, since the additional value $\theta$ remained still infinite. Therefore $\theta$ has to be discretized as well:

$$
\theta_{i}=\frac{\pi}{p} i, \quad \forall i=0,1, \ldots, 2 p-1, p \geq 2 .
$$

The number of discretization points of $\theta$ is restricted to even values.

With these discretizations the infinite problem becomes a finite one and can be stated as follows:

$$
\begin{aligned}
& \min _{\mathbf{h}} \mathbf{h}^{\mathrm{T}} \cdot \mathbf{D}_{N_{\mathbf{h}}} \cdot \mathbf{h} \\
& \text { s.t. } \mathbf{1}^{\mathrm{T}} \cdot \mathbf{h}=1 \text {, } \\
& \Omega_{0}:\left\{\begin{array}{c}
\mathbf{c}^{\mathrm{T}}\left(\Omega_{0}^{(\mathrm{i})}, \theta_{0}\right) \cdot \mathbf{h} \leq \Delta\left(\Omega_{0}^{(\mathrm{i})}\right) \\
\vdots \\
\mathbf{c}^{\mathrm{T}}\left(\Omega_{0}^{(\mathrm{i})}, \theta_{i}\right) \cdot \mathbf{h} \leq \Delta\left(\Omega_{0}^{(\mathrm{i})}\right) \\
\vdots \\
\mathbf{c}^{\mathrm{T}}\left(\Omega_{0}^{(\mathrm{i})}, \theta_{2 p-1}\right) \cdot \mathbf{h} \leq \Delta\left(\Omega_{0}^{(\mathrm{i})}\right)
\end{array}\right. \\
& \vdots \\
& \Omega_{L}:\left\{\begin{array}{c}
\mathbf{c}^{\mathrm{T}}\left(\Omega_{L}^{(\mathrm{i})}, \theta_{0}\right) \cdot \mathbf{h} \leq \Delta\left(\Omega_{L}^{(\mathrm{i})}\right) \\
\vdots \\
\mathbf{c}^{\mathrm{T}}\left(\Omega_{L}^{(\mathrm{i})}, \theta_{i}\right) \cdot \mathbf{h} \leq \Delta\left(\Omega_{L}^{(\mathrm{i})}\right) \\
\vdots \\
\mathbf{c}^{\mathrm{T}}\left(\Omega_{L}^{(\mathrm{i})}, \theta_{2 p-1}\right) \cdot \mathbf{h} \leq \Delta\left(\Omega_{L}^{(\mathrm{i})}\right) .
\end{array}\right.
\end{aligned}
$$


TABLe 1: Maximum Error over $p$.

\begin{tabular}{lccccc}
\hline$p$ & 2 & 4 & 8 & 16 & 32 \\
\hline$-20 \cdot \log (\cos (\pi / 2 \cdot p)) / \mathrm{dB}$ & 3.0103 & 0.6877 & 0.1685 & 0.0419 & 0.0105 \\
\hline
\end{tabular}

The price one has to pay for the linearization is the large number of inequality constraints in the stopband as pointed out in (55). The overall number of inequality constraints can be determined to $2 \cdot p \cdot L$.

The maximum error depends on factor $p$. The bigger $p$ is, the less the maximum error becomes. Table 1 shows the worst deviation from the constraints for some common values of $p$.

\section{Design of Low-Delay FIR Filter Bank Pair}

In this section a method to design a prototype filter for the SFB is introduced. The main objective lies in obtaining a distortion function of an oversampling I-channel complexmodulated filter bank according to (11) which independently of the frequency nearly equals a constant delay. At the same time the constant delay is supposed to be the smallest possible one as figured out in Section 2. Please note that all requirements regarding the distortion function are met on the synthesis filter bank side only. We use the deviation of the distortion function from a suitable desired distortion function as objective function, instead of minimizing the group delay of the distortion function, similar to minimizing the group delay of an FIR prototype filter in Section 3. The real-valued SFB prototype filter has to meet given magnitude frequency response specifications for the stopband. Due to the fact the constraints agree with those ones of the previous algorithm, the convex formulation in (51) can be used. Therefore only the objective function in (55) has to be modified.

4.1. Objective Function. In this section we present a convex objective function which minimizes the error between the distortion function and the desired distortion function during the optimization. In combination with the convex constraints in (51) it guarantees unique solutions.

The distortion function (16) depends on both AFB and SFB prototype filters as shown in Section 2. However the coefficients of the AFB prototype filter are regarded as constants in this design step, due to the fact they are fixed to the design result obtained in the first algorithm. Therefore the distortion function depends only on the SFB prototype filter: $F_{\text {dist }}\left(e^{\mathrm{j} \Omega^{(\mathrm{i})}}, \mathbf{g}\right)$. Below the dependence of the distortion function of $\mathbf{g}$ is pointed out only if required; otherwise we write $F_{\text {dist }}\left(\mathrm{e}^{\mathrm{j} \Omega^{(\mathrm{i})}}\right)$.

As discussed in Section 2 the group delay of the distortion function of oversampling complex-modulated filter banks is restricted to integral multiples of the number of channels $I$ only. For this reason the desired distortion function can be defined as follows:

$$
F_{\text {dist, desire }}\left(\mathrm{e}^{\mathrm{j} \Omega^{(\mathrm{i})}}\right)=\mathrm{e}^{-\mathrm{j} \kappa I \Omega^{(\mathrm{i})}},
$$

where $\kappa \in \mathbb{N}^{+}$. We are excluding the trivial case $\kappa=0$, since it is not realisable due to causality reasons [6]. By using the $L_{2}$-norm the objective function can be formulated as follows:

$$
\int_{-\pi}^{\pi}\left|F_{\text {dist }}\left(\mathrm{e}^{\mathrm{j} \Omega^{(\mathrm{i})}}, \mathbf{g}\right)-\mathrm{e}^{-\mathrm{j} \kappa I \Omega^{(\mathrm{i})}}\right|^{2} d \Omega^{(\mathrm{i})} .
$$

In order to obtain the lowest possible group delay, first the smallest possible $\kappa$ is selected, namely, $\kappa=1$. In case of dissatisfying results $\kappa$ is to increase gradually until the desired result is achieved.

4.2. Practical Implementation. Next we want to set up an objective function which can directly be implemented in numerical analysis programs like Matlab or Mathematica. To this end the integrand in (57) is reformulated in the following way:

$$
\begin{aligned}
& \left|F_{\text {dist }}\left(\mathrm{e}^{\mathrm{j} \Omega^{(\mathrm{i})}}\right)-\mathrm{e}^{-\mathrm{j} c \Omega^{(\mathrm{i})}}\right|^{2} \\
& \quad=\left(F_{\text {dist }}\left(\mathrm{e}^{\mathrm{j} \Omega^{(\mathrm{i})}}\right)-\mathrm{e}^{-\mathrm{j} \kappa I \Omega^{(\mathrm{i})}}\right) \cdot\left(F_{\text {dist }}^{*}\left(\mathrm{e}^{\mathrm{j} \Omega^{(\mathrm{i})}}\right)-\mathrm{e}^{\mathrm{j} \kappa I \Omega^{(\mathrm{i})}}\right) \\
& \quad=\left|F_{\text {dist }}\left(\mathrm{e}^{\mathrm{j} \Omega^{(\mathrm{i})}}\right)\right|^{2}-2 \operatorname{Re}\left\{\mathrm{e}^{\mathrm{j} c \Omega^{(\mathrm{i})}} \cdot F_{\text {dist }}\left(\mathrm{e}^{\mathrm{j} \Omega^{(\mathrm{i})}}\right)\right\}+1 .
\end{aligned}
$$

Reinserted in (57), we get an expression consisting of three separate integrals:

$$
\begin{aligned}
& \int_{-\pi}^{\pi}\left|F_{\text {dist }}\left(\mathrm{e}^{\mathrm{j} \Omega^{(\mathrm{i})}}\right)\right|^{2} d \Omega^{(\mathrm{i})} \\
& \quad-2 \int_{-\pi}^{\pi} \operatorname{Re}\left\{\mathrm{e}^{\mathrm{j} \kappa I \Omega^{(\mathrm{i})}} \cdot F_{\mathrm{dist}}\left(\mathrm{e}^{\mathrm{j} \Omega^{(\mathrm{i})}}\right)\right\} d \Omega^{(\mathrm{i})}+\underbrace{\int_{-\pi}^{\pi} d \Omega^{(\mathrm{i})}}_{2 \pi} .
\end{aligned}
$$

By applying Parseval's theorem on the left integral in (59) we get a formula which allows us to determine the value of the integral in time-domain [8]:

$$
\int_{-\pi}^{\pi}\left|F_{\text {dist }}\left(\mathrm{e}^{\mathrm{j} \Omega^{(\mathrm{i})}}, \mathbf{h}\right)\right|^{2} d \Omega^{(\mathrm{i})}=2 \pi \sum_{k=0}^{N_{\mathrm{s}}-1} f_{\text {dist }}^{2}(k)
$$

By inserting (15) into the right side of the upper expression the sum can be stated as follows:

$$
\int_{-\pi}^{\pi}\left|F_{\mathrm{dist}}\left(\mathrm{e}^{\mathrm{j} \Omega^{(\mathrm{i})}}, \mathbf{h}\right)\right|^{2} d \Omega^{(\mathrm{i})}=2 \pi \mathcal{O}^{2} \sum_{k=0}^{N_{\mathrm{s}}-1}\left[s_{0}^{(I)}(k)\right]^{2}
$$

Furthermore we omit all indices $k \neq m I$ since they are zero according to (15). The remaining sum is replaced by a 
weighted scalar product of two vectors:

$$
\begin{aligned}
\int_{-\pi}^{\pi}\left|F_{\text {dist }}\left(\mathrm{e}^{\mathrm{j} \Omega^{(\mathrm{i})}}, \mathbf{h}\right)\right|^{2} d \Omega^{(\mathrm{i})} & =2 \pi \mathcal{O}^{2} \sum_{m=0}^{\left\lfloor\left(N_{\mathrm{s}}-1\right) / I\right\rfloor} s^{2}(m I) \\
& =2 \pi \mathcal{O}^{2}\left(\mathbf{s}^{\mathrm{T}} \cdot \mathbf{s}\right) .
\end{aligned}
$$

The components of vector $\mathbf{s}$ consist of the convolution $s(k)=$ $h(k) * g(k)$ evaluated for the indices $m I, m=0, \ldots, \downarrow\left(N_{\mathrm{s}}-\right.$ $1) / I\rfloor$ as shown below:

$$
\mathbf{s}=\left(s(0), s(I), s(2 I), \ldots, s\left(\left\lfloor\frac{\left(N_{\mathrm{s}}-1\right)}{I}\right\rfloor I\right)\right)^{\mathrm{T}} .
$$

Let us have a closer look on $s(\kappa I)$ which according to (12) is

$$
s(m I)=\sum_{k=0}^{N_{\mathrm{g}}-1} h(m I-k) \cdot g(k) .
$$

Remember that the coefficients of the AFB prototype filter $h(k)$ are considered to be constants in the current step. Besides $h(k)$ is a causal FIR-filter (finite length), therefore $h(m I-k)$ in (12) is not equal to zero only if the following two conditions are fullfilled:

$$
\begin{gathered}
m I-k \geq 0 \Longrightarrow m I \geq k, \\
m I-k \leq N_{\mathrm{h}}-1 \Longrightarrow m I-N_{\mathrm{h}}+1 \leq k .
\end{gathered}
$$

Therefore all redundant zero-multiplications in $s(m I)$ are left out by taking the above inequations into account:

$$
s(m I)=\sum_{\max \left\{0, m I-N_{\mathrm{h}}+1\right\}}^{\min \left\{N_{\mathrm{g}}-1, m I\right\}} h(m I-n) \cdot g(n),
$$

and by applying the vector/matrix representation can be stated as follows:

$$
s(m I)=\mathbf{k}_{\mathrm{h}}^{\mathrm{T}}(m) \cdot \mathbf{g}
$$

The vector $\mathbf{k}_{\mathrm{h}}(m) \in \mathbb{R}^{N_{\mathrm{g}}}$ depends on the index $m$ and has the dimension $N_{\mathrm{g}}$. Its components are made up of $g(m I-$ $k$ ) for all indices $k$ which are included in the sum (66). The components which correspond to the remaining indices are simply put zero as shown below:

$$
\left[\mathbf{k}_{\mathrm{h}}(m)\right]_{k}= \begin{cases}g(m I-k), & \max \left\{0, m I-N_{\mathrm{h}}+1\right\} \\ & \leq k \leq \min \left\{N_{\mathrm{g}}-1, m I\right\}, \\ 0, & \text { otherwise. }\end{cases}
$$

Now the components $s(\kappa I)$ in (63) are replaced by using (67):

$$
\begin{aligned}
& \mathbf{s}=\left(\begin{array}{c}
s(0) \\
s(I) \\
s(2 I) \\
\vdots \\
s\left(\left\lfloor\frac{N_{s}-1}{I}\right\rfloor I\right)
\end{array}\right) \\
& =\underbrace{\left(\begin{array}{c}
\mathbf{k}_{\mathrm{h}}^{\mathrm{T}}(0) \\
\mathbf{k}_{\mathrm{h}}^{\mathrm{T}}(1) \\
\mathbf{k}_{\mathrm{h}}^{\mathrm{T}}(2) \\
\vdots \\
\mathbf{k}_{\mathrm{h}}^{\mathrm{T}}\left(\left\lfloor\frac{N_{\mathrm{s}}-2 / I}{I}\right\rfloor+1 \times N_{\mathbf{g}}\right.
\end{array}\right)}_{\mathbf{K} \in \mathbb{R}} \cdot \mathbf{g} .
\end{aligned}
$$

Vector $\mathbf{g}$ is pulled out as indicated above, and the remaining entries are combined to matrix $\mathbf{K}$ of dimension $\left\lfloor\left(N_{\mathrm{h}}+N_{\mathrm{g}}-\right.\right.$ $2) / I\rfloor+1 \times N_{\mathrm{g}}$. Please note that $\mathbf{K}$ consists only of inversed and shifted AFB coefficients $h(k)$. Its dimension depends on both the length of AFB and SFB prototype filters (i.e., $N_{\mathrm{h}}$ and $N_{\mathrm{g}}$ ) and the number of channels $I$.

Now vector $s$ in (62) is replaced by (69). We obtain a quadratic form in $\mathbf{g :}$

$$
\int_{-\pi}^{\pi}\left|F_{\text {dist }}\left(\mathrm{e}^{\mathrm{j} \Omega^{(\mathrm{i})}}, \mathbf{g}\right)\right|^{2} d \Omega^{(\mathrm{i})}=2 \pi \mathcal{O}^{2} \mathbf{g}^{\mathrm{T}} \cdot\left(\mathbf{K}^{\mathrm{T}} \cdot \mathbf{K}\right) \cdot \mathbf{g} .
$$

The second integral in (59) is

$$
2 \int_{-\pi}^{\pi} \operatorname{Re}\left\{\mathrm{e}^{\mathrm{j} \kappa I \Omega^{(\mathrm{i})}} \cdot F_{\text {dist }}\left(\mathrm{e}^{\mathrm{j} \Omega^{(\mathrm{i})}}\right)\right\} d \Omega^{(\mathrm{i})} .
$$

According to the inverse discrete-time Fourier transform of a discrete signal $x(k)$ evaluated explicitly for the zeroth coefficient [8]

$$
x(0)=\frac{1}{2 \pi} \int_{-\pi}^{\pi} X\left(\mathrm{e}^{\mathrm{j} \Omega^{(\mathrm{i})}}\right) \mathrm{e}^{\mathrm{j} \cdot 0 \cdot \Omega^{(\mathrm{i})}} d \Omega^{(\mathrm{i})},
$$

the integral in (71) formally corresponds to

$$
4 \pi \cdot x(0)=2 \int_{-\pi}^{\pi} X\left(\mathrm{e}^{\mathrm{j} \Omega^{(\mathrm{i})}}\right) d \Omega^{(\mathrm{i})} .
$$

Therefore the evaluation of the integral in (71) is reduced to the determination of the zeroth coefficient of the inverse discrete-time Fourier transform of the following expression:

$$
\operatorname{Re}\left\{\mathrm{e}^{\mathrm{j} \kappa I \Omega^{(\mathrm{i})}} \cdot F_{\text {dist }}\left(\mathrm{e}^{\mathrm{j} \Omega^{(\mathrm{i})}}\right)\right\} .
$$

The inverse discrete-time Fourier transform of (74) can be obtained by first applying the time-shift property of the discrete-time Fourier transform [8]:

$$
x\left(n-n_{0}\right) \stackrel{\text { DTFT }}{\longleftrightarrow} \mathrm{e}^{-\mathrm{j} n_{0} \Omega^{(\mathrm{i})}} X\left(\mathrm{e}^{\mathrm{j} \Omega^{(\mathrm{i})}}\right),
$$


and secondly using the fact that in case of real-valued signals the real part in frequency domain corresponds to the even part in the time-domain [8]:

$$
\frac{1}{2}[x(n)+x(-n)] \stackrel{\mathrm{DTFT}}{\longleftrightarrow} \operatorname{Re}\left\{X\left(\mathrm{e}^{\mathrm{j} \Omega^{(\mathrm{i})}}\right)\right\}
$$

When applied on (74) we get

$$
\begin{aligned}
& \frac{1}{2}\left[f_{\text {dist }}(n+\kappa I)+f_{\text {dist }}(-n+\kappa I)\right] \\
& \stackrel{\text { DTFT }}{\longleftrightarrow} \operatorname{Re}\left\{\mathrm{e}^{\mathrm{j} \kappa I \Omega^{(\mathrm{i})}} \cdot F_{\text {dist }}\left(\mathrm{e}^{\mathrm{j} \Omega^{(\mathrm{i})}}, \mathbf{g}\right)\right\} .
\end{aligned}
$$

Next we use (15) to express the distortion function in the time-domain as a function of the SFB prototype filter coefficients. Therefore the zeroth coefficient of (74) is

$$
f_{\text {dist }}(\kappa I)=\mathcal{O} \cdot s_{0}^{(I)}(\kappa I) .
$$

The upper term can be simplified according to (15). When (78) is inserted in (73), we get an expression for the second integral:

$$
4 \pi \cdot \mathcal{O} \cdot s(\kappa I)=2 \int_{-\pi}^{\pi} \operatorname{Re}\left\{\mathrm{e}^{\mathrm{j} \kappa I \Omega^{(\mathrm{i})}} \cdot F_{\text {dist }}\left(\mathrm{e}^{\mathrm{j} \Omega^{(\mathrm{i})}}, \mathbf{g}\right)\right\}^{2} d \Omega^{(\mathrm{i})}
$$

Finally the second integral according to (79) is written by using (67):

$$
4 \pi \mathcal{O} \cdot \mathbf{k}_{\mathrm{h}}^{\mathrm{T}}(\kappa) \cdot \mathbf{g}=2 \int_{-\pi}^{\pi} \operatorname{Re}\left\{\mathrm{e}^{\mathrm{j} \kappa I \Omega^{(\mathrm{i})}} \cdot F_{\text {dist }}\left(\mathrm{e}^{\mathrm{j} \Omega^{(\mathrm{i})}}, \mathbf{g}\right)\right\}^{2} d \Omega^{(\mathrm{i})} .
$$

The convex objective function in (57) is readily formulated as a quadratic function in $\mathbf{g}$ :

$$
\begin{aligned}
& \int_{-\pi}^{\pi}\left|F_{\text {dist }}\left(\mathrm{e}^{\mathrm{j} \Omega^{(\mathrm{i})}}, \mathbf{g}\right)-\mathrm{e}^{-\mathrm{j} c \Omega^{(\mathrm{i})}}\right|^{2} d \Omega^{(\mathrm{i})} \\
& \quad=2 \pi \mathcal{O}^{2} \mathbf{g}^{\mathrm{T}} \cdot\left(\mathbf{K}^{\mathrm{T}} \cdot \mathbf{K}\right) \cdot \mathbf{g}-4 \pi \cdot \mathcal{O} \cdot \mathbf{k}_{\mathrm{h}}^{\mathrm{T}}(m) \cdot \mathbf{g}+2 \pi .
\end{aligned}
$$

Please note that since matrix $\mathbf{K}$ only depends on the coefficients, $\mathbf{h}$ is has to be computed only once. It remains unchanged during the iterations.

\section{Design Example}

Subsequently, we present an example for the design of a uniform oversampling complex-modulated I-channel FBP, where $I=64$. The decimation factor is $M=16$, resulting in an oversampling factor of $\mathcal{O}=4$.

5.1. AFB Prototype Filter. First we start with the design of a narrow-band FIR low-pass AFB prototype filter with low group delay designed by using the algorithm described in Section 3. For the implementation of the design algorithm we used the built-in function fmincon of the Optimization

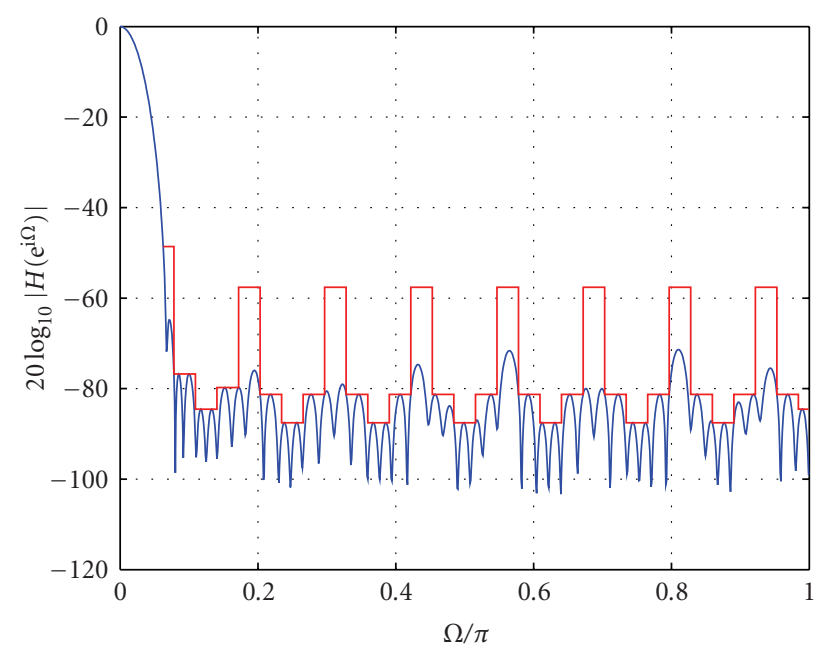

(a) Logarithmic magnitude frequency response

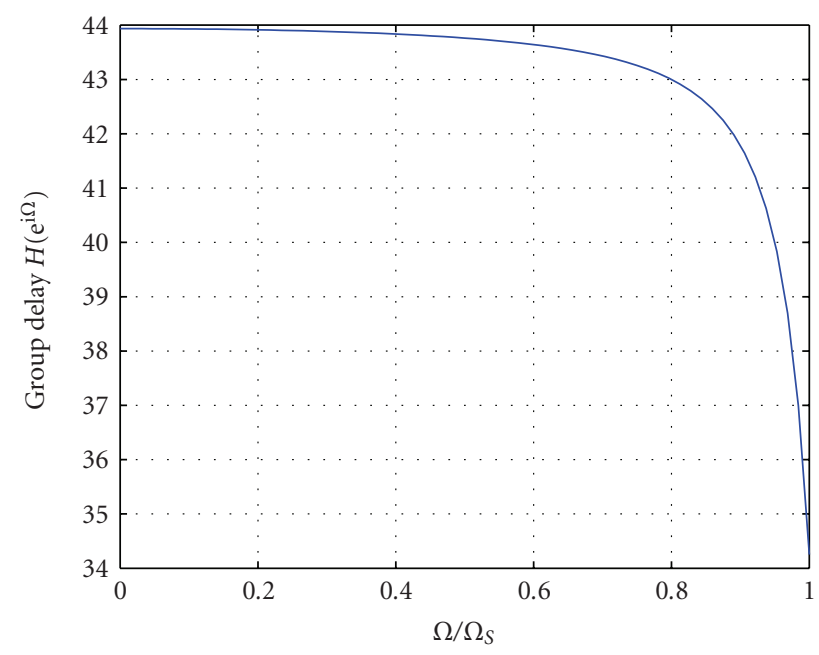

(b) Group delay AFB

Figure 2: Narrow-band FIR low-pass filter.

Toolbox for Matlab. The magnitude frequency response specifications for the stopband are chosen according to the considerations made in [7]. The minimum possible filter length in order to fulfill the given magnitude specifications turned out to be $N_{\mathrm{h}}=90$. The number of frequency points $L$ in (55) was chosen to be 1024 . The maximum number $p$ of the point of the rotation factor $\theta$ in (54) was chosen to be 32 thus according to Table 1 producing a maximum error of $0.0105 \mathrm{~dB}$.

The logarithmic magnitude frequency response along with the tolerance mask for the stopband defined in [7] is depicted in Figure 2(a). We notice that the tolerance mask is not always touched by the magnitude response. In some regions the magnitude response ranges far below the allowed attenuation, which can be traced back to the fact that the tolerance mask is not continuous and increases and diminishes stepwise.

Figure 2(b) depicts the group delay both in passband and transition band. The group delay in the passband, which 


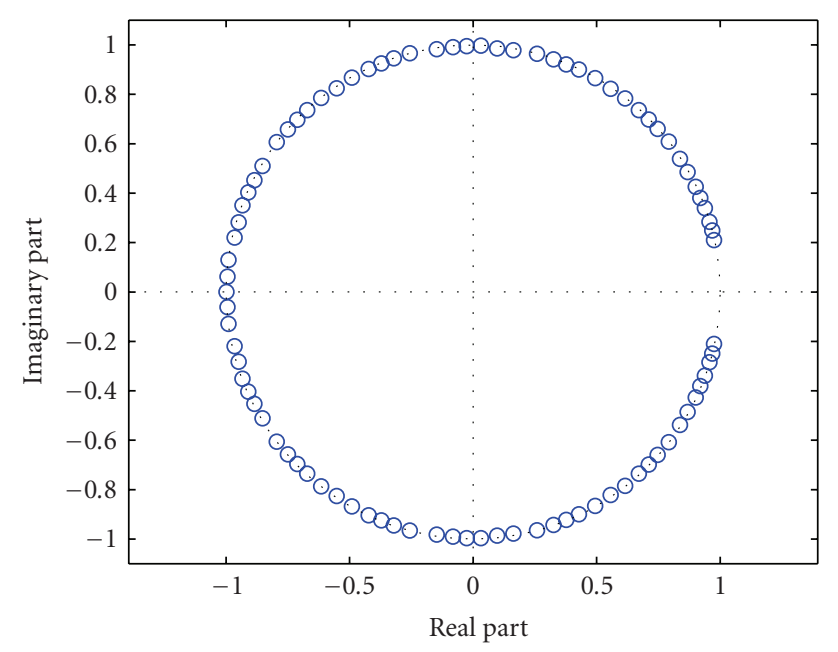

Figure 3: Zero plot of $H(z)$.

ranges from the normalized frequency $\Omega / \Omega_{\mathrm{s}}=0$ up to $\Omega / \Omega_{\mathrm{s}}=0.25$, is almost constant with the mean value of $\tau_{\mathrm{g}, \mathrm{AFB}}=43.9$. In the transition band, which starts at the normalized frequency $\Omega / \Omega_{\mathrm{s}}=0.25$, the group delay features a decay similar to that of the magnitude response. The group delay however does not fall below the value of $\tau_{\mathrm{g}, \mathrm{AFB}}=43 \mathrm{up}$ to the normalized frequency $\Omega / \Omega_{\mathrm{s}}=0.8$ such that the group delay can be regarded as constant in the region, where the magnitude frequency response does not range below $40 \mathrm{~dB}$.

As to be seen from Figure 3 there are no zeros forming the passband, which is common for narrow-band filters, since no distinct passband is existent here. All zeros are effectuating the stopband. They are distributed on the periphery of the unit circle. However they are located slightly inside the $z$-plane unit circle, thus yielding a minimum-phase filter. Moreover, zeros within the unit circle contribute to the reduction of the overall group delay of the passband [8].

5.2. SFB Prototype Filter. To complete the FBP design we present an example for the narrow-band FIR low-pass SFB prototype filter designed by the procedure in Section 4 . This SFB prototype filter is matched to the AFB prototype filter of Section 5.1 such that the distortion function of an oversampling $I$-channel complex-modulated FIR filter bank according to (11) approximates a constant delay (LP allpass function). At the same time the constant delay is supposed to be the smallest possible one as figured out in Section 2.

The magnitude response specifications of the stopband are chosen according to the considerations made in [7]. They are more strict than those of the AFB for reasons stated in [7]. For the implementation of the design algorithm the built-in function fmincon of the Optimization Toolbox of Matlab is used again. The given stopband magnitude specifications are met for the filter length $N_{\mathrm{g}}=152$. The number of frequency points is again $L=1024$.

Figure 4(a) shows the logarithmic magnitude frequency response. The tolerance mask for the stopband is depicted

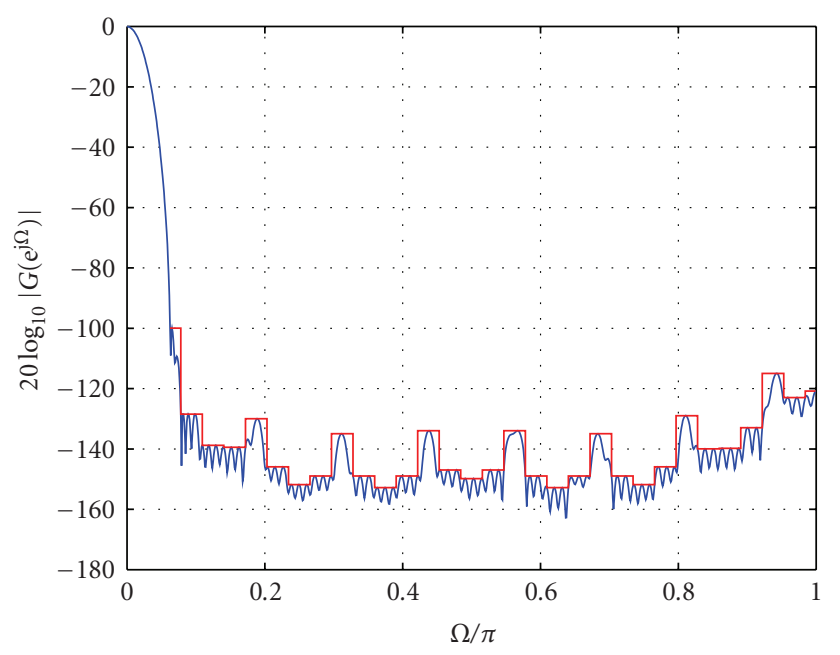

(a) Logarithmic magnitude response SFB

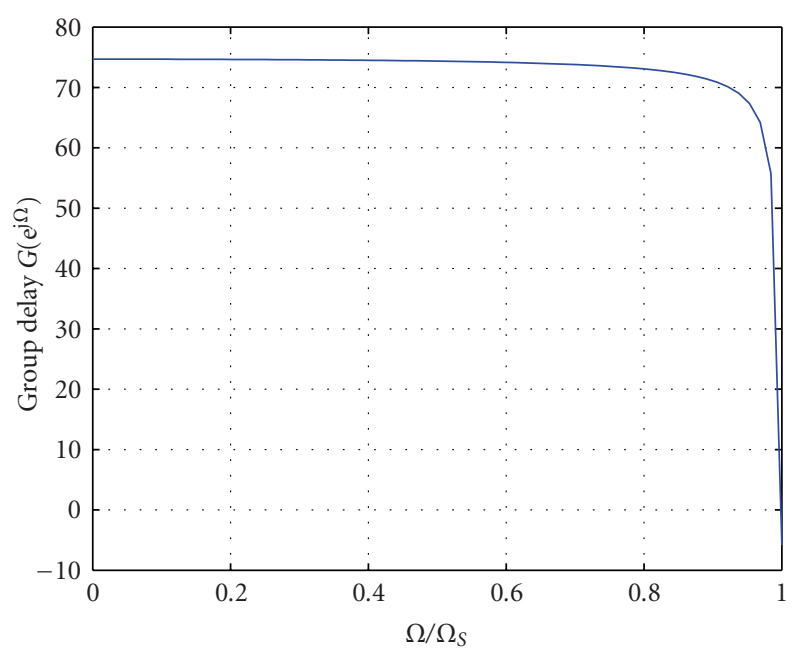

(b) Group delay SFB

FIgURE 4: Narrow-band SFB prototype filter.

in red color. We notice that this time the tolerance mask is always touched by the magnitude response. This can be traced back to the fact that, for the SFB, the steps of the tolerance mask are much smaller, not exceeding $20 \mathrm{~dB}$, except for the region next to the transition band.

The group delay in the passband and transition band is depicted in Figure 4(b). The group delay is again approximately constant with the mean value $\tau_{\mathrm{g}, \mathrm{SFB}}=74$. This time the group delay does not decay below the value of $\tau_{\mathrm{g}, \mathrm{SFB}}=70$ roughly up to the frequency where the magnitude response is $80 \mathrm{~dB}$. The obtained SFB prototype filter is again minimum-phase, even though no demand is made in this regard according to Section 4 .

Figure 5(a) illustrates the logarithmic magnitude response of the distortion function. It approximates the constant value of zero $\mathrm{dB}$ with a peak-to-peak deviation amounting to $1.2 \mathrm{~dB}$, which lies within the tolerance limits defined in [7]. Moreover the magnitude response exhibits periodicity, as described in Section 2. 


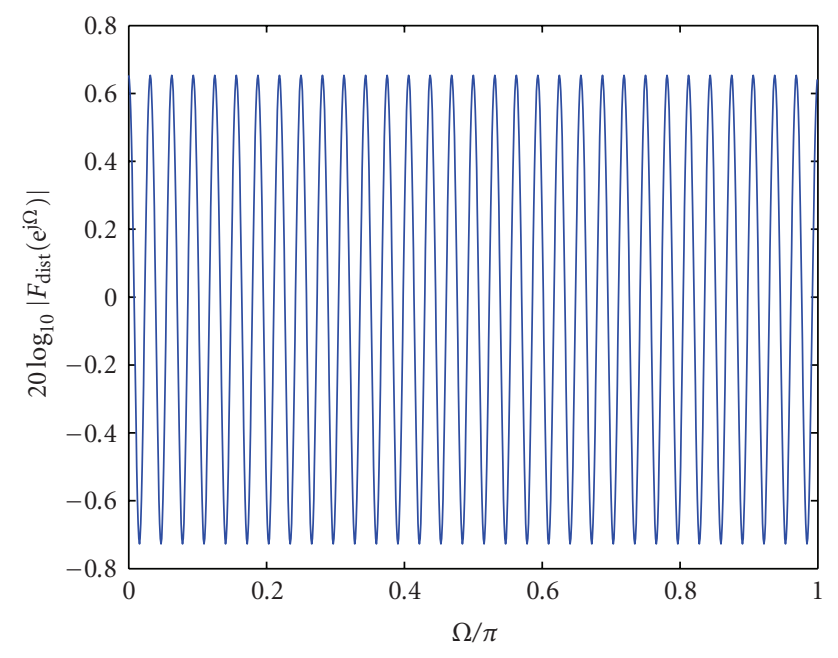

(a) Logarithmic magnitude response of the distortion function.

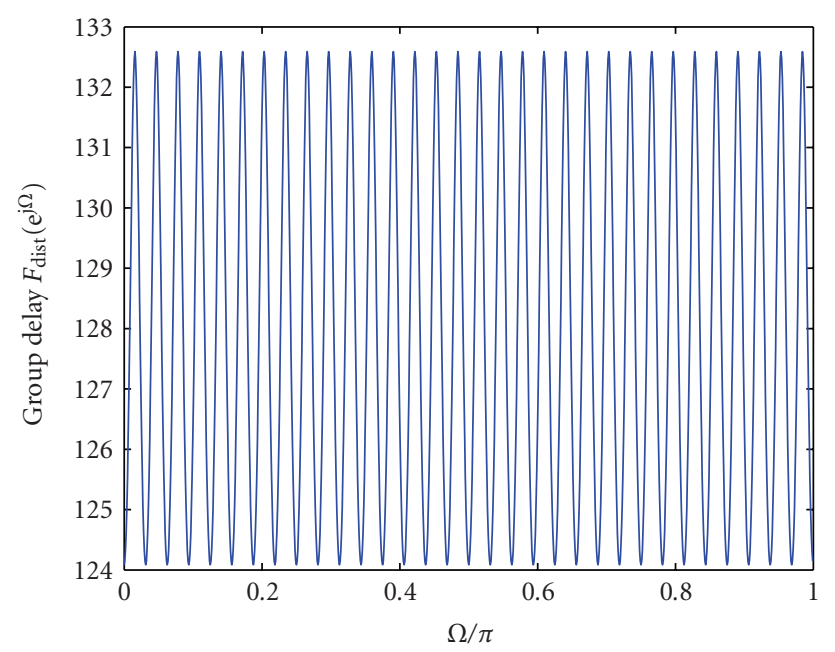

(b) Group delay of the distortion function.

FIgURE 5: Distortion Function.

The group delay of the distortion function is depicted in Figure 5(b). It varies around the feasible value of $2 I=128$ derived in Section 2.3 with a deviation of \pm 4 corresponding to $\pm 3 \%$.

The downsampled FBP impulse response $f_{\text {dist }}(m I)$ in compliance with (15) is depicted in Figure 6 (here all zero coefficients have been omitted). The number $\left\lfloor\left(N_{s}-1\right) / I\right\rfloor+$ 1 of non-zero coefficients is four, since $N_{\mathrm{s}}$ according to (13) results in $N_{s}=241$. Here the dominating coefficient is the second one, thus explaining the overall delay of 128 as shown above. Note that, due to the even number of non-zero coefficients, no exact linear-phase distortion function is possible. The only way to approximate an allpass according to the considerations in Section 2 is by bringing the coefficients as close as possible to a ideal delay.



FIGURE 6: Distortion function in time-domain $f_{\text {dist }}(m I)$.

\section{Conclusion}

In this contribution, a new iterative approach for the design of analysis and synthesis prototype filters of oversampling uniform complex-modulated FIR filter bank pairs is proposed, such that the overall FBP signal delay is minimized, and the subband signals experience short delay.

We have shown that in the time-domain the distortion function can be expressed as a simple FIR filter impulse response. Based on these results we have shown that the mean value of the group delay is restricted to integer multiples of the number of channels.

For the first design step we have introduced a novel procedure for the design of low-delay AFB FIR prototype filters with approximately linear-phase in the passband and the transition band. This procedure is based on convex constrained optimization which guarantees unique solutions. To this purpose we have introduced a convex objective function for group delay minimisation, which is based on a particular representation of the group delay according to [20]. The magnitude requirements are used as design constraints. The magnitude specifications (e.g., for hearing aids those derived in [7]) serve as stopband constraints to control aliasing.

For the second and final design step, we have presented a procedure for the design of the SFB FIR prototype filter, such that the overall signal delay of the FBP is minimized. This procedure is again based on convex constrained optimization, where in analogy to the first design step the magnitude specifications serve as stopband constraints to control imaging. Based on the theoretical results regarding the minimal feasible delays, the objective function is chosen as the deviation of the FBP distortion function from a prescribed $I$-fold delay. In this step, the AFB prototype filter is fixed to the design result obtained in the previous step. Furthermore we have presented an efficient implementation of the objective function to cope with high computational load. 
Finally, we have discussed the properties of the design algorithm with reference to an example. The example shows that the group delays of the prototype filters obtained using the presented procedures exhibit almost constant group delay not only in the passband but also in the transition band. The mean value of the group delay ranges below that of linear-phase filters of the same length. The observed overall signal delay lies within the tolerances defined in [7] and approximates the feasible delay as described in Section 2.

A comparison between the proposed design method against several other approaches to the design of oversampling complex-modulated FBS is not performed here, since [22] treats this topic thoroughly, where approaches by DAM et al. [15], by Stöcker et al. [23], and by Bäuml and Sörgel [24] are compared.

Future investigations will be devoted to the application of the prototype filter pair presented in this contribution to uniform, complex-modulated filter banks with additional subband signal manipulation. We will particularly investigate whether or not amplification of subband signals has an impact on the group delay of the distortion function.

\section{References}

[1] V. Harnacher, J. Chalupper, J. Eggers, et al., "Signal processing in high-end hearing aids: state of the art, challenges, and future trends," EURASIP Journal on Applied Signal Processing, vol. 2005, no. 18, pp. 2915-2929, 2005.

[2] M. A. Stone and B. C. J. Moore, "Tolerable hearing aid delays. I. Estimation of limits imposed by the auditory path alone using simulated hearing losses," Ear \& Hearing, vol. 20, no. 3, pp. 182-192, 1999.

[3] M. A. Stone and B. C. J. Moore, "Tolerable hearing aid delays. III. Effects on speech production and perception of acrossfrequency variation in delay," Ear \& Hearing, vol. 24, no. 2, pp. 175-183, 2003.

[4] P. Vary, Ein Beitrag zur Kurzzeitspektralanalyse mit digitalen Systemen, Ph.D. dissertation, Universität Erlangen-Nürnberg, Erlangen, Germany, 1978.

[5] P. P. Vaidyanathan, Multirate Systems and Filter Banks, Prentice-Hall, Englewood Cliffs, NJ, USA, 1993.

[6] H. G. Göckler and A. Groth, Multiratensysteme, Schlembach Fachverlag, Willburgstetten, Germany, 2004.

[7] D. Alfsmann, H. G. Göckler, and T. Kurbiel, "FrequencyDomain Magnitude Constraints for Oversampling ComplexModulated NPR Filter Bank System Design Ensuring a Prescribed Signal-to-Distortion Ratio," to be published.

[8] A. V. Oppenheim and R. W. Schafer, Discrete-Time Signal Processing, Prentice-Hall, London, UK, 1989.

[9] T. Kurbiel, D. Alfsmann, and H. G. Göckler, "Design of highly selective quasi-equiripple FIR lowpass filters with approximately linear phase and very low group delay," in Proceedings of the European Signal Processing Conference (EUSIPCO '08), EURASIP, Lausanne, Switzerland, 2008.

[10] T. F. Liau, M. A. Razzak, and L. G. Cuthbert, "Phase constraints on fir digital filters," Electronics Letters, vol. 17, no. 24, pp. 910-911, 1981.

[11] H. Baher, "Fir digital filters with simultaneous conditions on amplitude and delay," Electronics Letters, vol. 18, no. 7, pp. 296-297, 1982.
[12] E. Sharestani and L. G. Cuthbert, "Fir digital filters designed to a groupdelay characteristic," Electronics Letters, vol. 21, no. 12, pp. 542-544, 1985.

[13] X. Zhang and T. Yoshikawa, "Design of FIR Nyquist filters with low group delay," IEEE Transactions on Signal Processing, vol. 47, no. 5, pp. 1454-1458, 1999.

[14] M. Lang, Algorithms for the constrained design of digital filters with arbitrary magnitude and phase responses, $\mathrm{Ph}$.D. dissertation, Vienna University of Technology, Vienna, Austria, June 1999.

[15] H. H. Dam, S. Nordholm, A. Cantoni, and J. M. de Haan, "Iterative method for the design of DFT filter bank," IEEE Transactions on Circuits and Systems II, vol. 51, no. 11, pp. 581586, 2004.

[16] H. H. Dam, S. Nordholm, and A. Cantoni, "Uniform FIR filterbank optimization with group delay specifications," IEEE Transactions on Signal Processing, vol. 53, no. 11, pp. 42494260, 2005.

[17] W. Kellermann, "Analysis and design of multirate systems for cancellation of acoustic echoes," in Proceedings of IEEE International Conference on Acoustics, Speech and Signal Processing (ICASSP '88), pp. 2570-2573, 1988.

[18] K. F. C. Yiu, N. Grbic, S. Nordholm, and K. L. Teo, "Multicriteria design of oversampled uniform DFT filter banks," IEEE Signal Processing Letters, vol. 11, no. 6, pp. 541-544, 2004.

[19] K. F. C. Yiu, N. Grbic, S. Nordholm, and K. L. Teo, "A hybrid method for the design of oversampled uniform DFT filter banks," Signal Processing, vol. 86, no. 7, pp. 1355-1364, 2006.

[20] H. W. Schüßler, Digitale Signalverarbeitung 1, Springer, Heidelberg, Germany, 5th edition, 2008.

[21] T. W. Parks and C. S. Burrus, Digital Filter Design, John Wiley \& Sons, New York, NY, USA, 1987.

[22] D. Alfsmann, H. G. Göckler, and T. Kurbiel, "Filter banks for hearing aids applying subband amplification: a comparison of different specification and design approaches," in Proceedings of the 17th European Signal Processing Conference (EUSIPCO '09), Glasgow, Scotland, August 2009.

[23] C. Stöcker, T. Kurbiel, D. Alfsmann, and H. G. Göckler, "A novel approach to the design of oversampling complexmodulated digital filter banks," in Proceedings of the 17th European Signal Processing Conference (EUSIPCO '09), Glasgow, Scotland, August 2009.

[24] R. W. Bäuml and W. Sörgel, "Uniform polyphase filter banks for use in hearing aids: design and constraints," in Proceedings of the European Signal Processing Conference (EUSIPCO '08), EURASIP, Lausanne, Switzerland, 2008. 